\title{
Placenta Increta at 14 Weeks with Subsequent Hysterectomy: A Case Report
}

\author{
Abigail E Collett ${ }^{1 *}$, Jean Payer ${ }^{1}$ and Xuezhi Jiang ${ }^{1,2}$ \\ ${ }^{1}$ Departments of OB/GYN, The Reading Hospital of Tower Health System, Reading, USA \\ ${ }^{2}$ Departments of OB/GYN, Sidney Kimmel Medical College of Thomas Jefferson University, Philadelphia, USA
}

*Corresponding author: Abigail E Collett, DO., Department of OB/GYN-R1, The Reading Hospital of Tower Health, P.O. Box: 16052, Reading, Philadelphia, PA 19612-6052, USA, Tel: 484-628-8827, Fax: 484-628-9292, E-mail: Abigail.collett@ towerhealth.org

\begin{abstract}
Background: Abnormal placental implantation (API) is an uncommon obstetrical phenomenon that is associated with significant maternal morbidity. Typically, the diagnosis of API is made at the time of delivery in the third trimester. However there are reports of API presenting in the second trimester, and rarely in the first trimester. Cesarean Scar Pregnancy (CSP) can be considered a subset of API. Early $\mathrm{API}$ rarely occurs in the absence of previous uterine manipulation. CSP is always associated with previous cesarean delivery. Nonetheless, it has been suggested that CSP and early API are histologically indistinguishable and both are precursors of API.
\end{abstract}

Case Report: A 38 yo G4P2012 with a history of two previous cesarean sections underwent a dilation curettage and evacuation (DC\&E) procedure at $14 w 6 d$ gestational age (GA) dated by last menstrual period (LMP) after approximately one month of expectant management for an incomplete spontaneous abortion. Beta-hCG on the morning of surgery was $1,936 \mathrm{mIU} / \mathrm{mL}$. In the operating room (OR), immediately after the bimanual exam was performed heavy bright red vaginal bleeding was noted to be coming from the cervical os. A steady heavy flow of bleeding continued throughout the procedure. Intra-operatively the patient became hypotensive and was transfused with two units of packed red blood cells. Uterotonic agents were administered and a Bakri balloon was placed to tamponade the bleeding. At the conclusion of the case no further active bleeding was noted and the patient was hemodynamically stable. Approximately 7-8 hours after the initial DC\&E procedure the patient bled around the Bakri balloon and was noted to be tachycardic. About nine hours after the initial surgery the decision was made to return to the OR due to persistent vaginal bleeding, persistent tachycardia, additional blood product requirements, and re-review of early transvaginal ultrasound which showed heterogeneous myometrium and a heterogeneous mass-like solid and cystic appearing area in the lower uterine segment. A total abdominal hysterectomy, bilateral salpingectomy and cystoscopy were performed. Intra-operative evaluation revealed a firm protruding tissue mass in the left anterior lower uterine segment that was found to be placenta increta or more on final pathology. The patient recovered appropriately during her hospital stay and was discharged in stable condition.

Conclusion: API is an uncommon finding in early pregnancy. Although there are few reported cases, the majority of documented cases presented similarly to our case. Although specific screening for API or CSP is not recommended, it is important to have a high index of suspicion and proper preparation for patients with multiple risk factors for API, especially previous cesarean sections. It is important to consider early API and CSP as differential diagnoses when one encounters unexplainable and unexpected hemorrhage at the time of DC\&E procedure.

\section{Keywords}

Abnormal placenta implantation, Placenta increta, Early second trimester pregnancy, Hysterectomy, Cesarean scar pregnancy

\section{Introduction}

Abnormal placental implantation (API) occurs in approximately 1 in 533 deliveries in the USA and is associated with significant maternal morbidity [1]. There are varying degrees of abnormal implantation differentiated by depth of placental invasion. In order of increasing severity, placenta accreta is invasion into the myometrium, increta is invasion deeper into the myometrium, and percreta is invasion through the myometrium and 
serosa and potentially into surrounding structures. API occurs in areas where there is a total or partial defect in the decidua basalis of the endometrium. Injury to the myometrium causes scarring. As the myometrium heals it forms a fibrous collagen layer rather than regenerating muscle [1]. The scarred area is weaker and does not form functional decidua basalis, which increases the risk of abnormal placentation [2]. There are various proposed theories to explain exactly how and why API occurs. These include: A primary defect in trophoblast function, secondary decidua basalis defect due to failure of normal decidua formation, and finally abnormal vascularization and oxygenation of the scarred tissue [1].

Risk factors for API include previous cesarean section, curettage procedure, placenta previa with a history of cesarean section, manual removal of placenta, endometritis, myomectomy, Asherman's syndrome, submucosal fibroids, adenomyosis, increasing age, and multiparity [1-3]. Studies show that cesarean section is the most important risk factor associated with API and the risk increases with increasing number of prior cesarean sections [1]. Despite API typically being associated with previous uterine injury or a risk factor that is associated with a weakening of the endometrium, it has been reported that about $7 \%$ of API occur in women without risk factors [3].

Most cases of API are documented in the third trimester or at the time of delivery. Less commonly there are reports of API presenting in the second trimester, often associated with post-abortal hemorrhage and less commonly uterine rupture [3]. API is an uncommon finding in the first trimester. Papadakis, et al. did a MEDLINE search using key words "placenta accreta", "placenta percreta", "early gestation" and "pregnancy" from 1966 to 2007 which showed only 14 histologically proven cases of first trimester API [3]. Most of these cases presented with severe hemorrhage precipitated by curettage for induced or spontaneous abortion. All but one case resulted in definitive management with total abdominal hysterectomy (TAH). The one outlier was treated with bilateral uterine artery embolization (UAE). Rouholamin, et al. published two cases documenting similar presentations; patients with history of cesarean sections undergoing curettage procedure for first trimester loss, resulting in severe bleeding and ultimately requiring total abdominal hysterectomy [4].

Cesarean scar pregnancy (CSP) is another uncommon complication seen in patients with prior cesarean sections and is also associated with significant maternal morbidity. The rate of CSP is increasing with the increased rate of cesarean deliveries, now affecting approximately 1 in 800-2500 women [5]. Timor-Tritsch, et al. did a retrospective review of histopathological features of patients with a diagnosis of CSP and early abnormal placentation (early second trimester API). Their study showed that these two entities are histologically

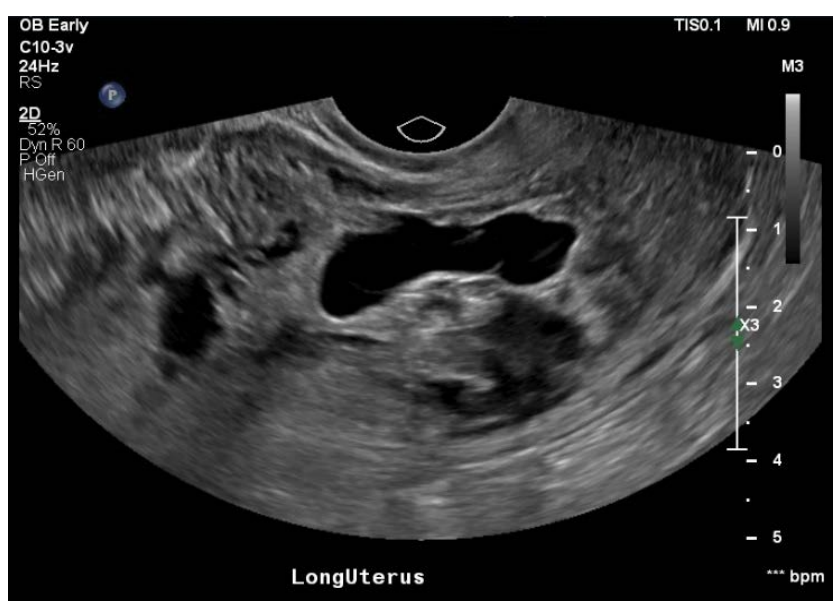

Figure 1: Transvaginal dating ultrasound prior to initial obstetrical visit.

indistinguishable, but are both precursors to API.

\section{Case}

A 38 yo G4P2012 with a past medical history of hypothyroidism presented for an initial obstetric (OB) appointment to an outside institution. Obstetrical history included two previous cesarean sections and one previous spontaneous abortion. The patient underwent transvaginal ultrasound (TVUS) the day prior to her visit at 10w0d gestational age (GA) based off last menstrual period (LMP) (Figure 1). The ultrasound showed an elongated sac-like focus that did not have the appearance of a normal intrauterine gestational sac. The ultrasound also did not show a definite yolk sac or fetal pole. The radiology report pointed out a heterogeneous myometrium and a heterogeneous mass-like solid and cystic appearing area approximately $5.4 \mathrm{~cm}$ in the lower uterine segment. Per the report these findings were non-specific but may reflect a fibroid, however other mass lesions could not be excluded with certainty. Molar pregnancy was considered less likely.

At her initial OB appointment, the patient reported menses-like vaginal bleeding for around one week. Per the patient she elected for expectant management and a referral was made to the clinic at our institution for a possible dilation, curettage and evacuation (DC\&E) procedure. The next documented patient encounter was in our facility's obstetrical triage department at $14 \mathrm{w} 2 \mathrm{~d}$, about one month after her initial presentation. The patient presented with abdominal cramping and persistent vaginal bleeding. Review of serum quantitative beta-hCG $(\mathrm{mlU} / \mathrm{mL})$ showed a downward trend consistent with spontaneous abortion: 2/28/2018: 10,181, 3/9/2018: 5,727, 3/25/2018: 1,854. Repeat TVUS in triage was consistent with the prior diagnosis, showing an irregular shape and elongated gestational sac and no yolk sac or fetal pole. The patient was seen in the clinic two days later where she was re-evaluated and consented for a DC\&E procedure and blood transfusion.

She presented for the procedure two days later at 
$14 \mathrm{w} 6 \mathrm{~d}$. She had a complete blood count (CBC) and type and screen prior to the procedure. Beta-hCG on the morning of surgery was $1,936 \mathrm{mlU} / \mathrm{mL}$. In the operating room (OR), she was placed under MAC anesthesia. Bimanual exam revealed an approximately 8 week size, mobile, anteverted uterus, no adnexal masses and the cervix was one centimeter dilated. Immediately after the bimanual exam heavy bright red vaginal bleeding was noted to be coming from the cervical os. A steady heavy flow of bright red blood continued during the suction curettage procedure. A large amount of necrotic tissue was removed with curettage and sent to pathology. There continued to be a heavy steady flow of bright red blood. A Foley balloon was inserted into the fundus and inflated with $20 \mathrm{cc}$ normal saline, but bleeding continued to flow around the catheter. The Foley balloon was removed. Bleeding slightly slowed, but was still actively flowing from the os. A Bakri balloon was then placed and inflated with approximately 150 cc of saline. No further bleeding was noted around the Bakri or through the Bakri balloon tubing. She was monitored intra-operatively for about fifteen minutes and a minimal amount of blood was observed in the Bakri balloon tubing at the conclusion of the case. Due to the large volume of blood loss (estimated blood loss 1500 cc) the patient became hypotensive. An intra-operative hemoglobin was 9.2 (pre-operative 11.5), so one unit of packed red blood cells (PRBC) was transfused. Pressors were administered, and she received $250 \mathrm{mg}$ albumin. She was given uterotonic agents intra-operatively; 0.2 $\mathrm{mg}$ IM methergine and $1000 \mathrm{mcg}$ misoprostol per rectum.

The patient remained hemodynamically stable in the PACU and received 2 additional units of PRBC and 1 unit of fresh frozen plasma (FFP). She was subsequently transferred to the Surgical ICU for close monitoring. At her post-operative evaluation there was bleeding noted around the Bakri and $250 \mathrm{cc}$ blood collected in Bakri bag over approximately 4 hours. A Pitocin bolus was administered and a Pitocin infusion was initiated at $125 \mathrm{cc} /$ hour. Methergine $0.2 \mathrm{mg}$ IM was also administered and scheduled for every eight hours. Two hours later she was re-evaluated and bleeding was still noted to be flowing around the Bakri. Examination revealed the Bakri balloon at the introitus. The Bakri balloon was removed and a bimanual exam revealed a $4 \mathrm{~cm}$ dilated cervix and about $250-300 \mathrm{cc}$ of blood clot was removed. The Bakri was replaced and inflated again with $150 \mathrm{cc}$ of saline. She was given another two units of PRBC, one unit of FFP and one unit of platelets. About an hour later the patient was re-evaluated. An additional $250 \mathrm{cc}$ blood was noted in the Bakri and her pad was saturated. New onset of tachycardia was also noted at the time of this exam. One hour later continuous bleeding was noted and on exam the Bakri was again found in the vagina. Another bimanual exam was performed which showed a dilated and floppy lower uterine segment with non- palpable uterine fundus. The patient continued to be tachycardic. Given the continued bleeding, abnormal vital signs and re-review of the abnormal appearing initial dating ultrasound, molar pregnancy was suspected and the patient was not felt to be a candidate for uterine artery embolization (UAE). Instead, the patient was consented for an exploratory laparotomy, total abdominal hysterectomy (TAH), bilateral salpingectomy (BS) and cystoscopy.

The patient returned to the OR where TAH, BS, cystoscopy were performed. Findings from a pre-operative bimanual exam under anesthesia revealed the following: External genitalia with persistent bleeding from vagina, extraction of clot, non-palpable uterus, cervix dilated to $3 \mathrm{~cm}$. No lacerations, tears or active bleeding visualized during thorough examination of the vagina and cervix. Findings at the time of the TAH, BS, and cystoscopy were as follows: Multiple adhesions to the anterior abdominal wall, omental adhesions to bladder and lower uterine segment, the bladder was adherent to lower uterine segment, bilateral fallopian tubes and ovaries were normal appearing, the fundus was firm and small, and the lower uterine segment was floppy and dilated. After dense bladder adhesions were lysed and the bladder was dissected off the lower uterine segment there was a firm tissue mass about $3-4 \mathrm{~cm}$ in size visualized protruding from the left lower uterine segment (Figure 2). This area was suspicious for placenta accreta vs. abnormal implantation site. Cystoscopy revealed a normal dome and trigone, brisk bilateral efflux of urine from bilateral ureteral orifices, and no visualized sutures or damage to the bladder.

Intra-operatively she received an additional one unit of FFP, two units PRBC, and 250 cc albumin. EBL was 350

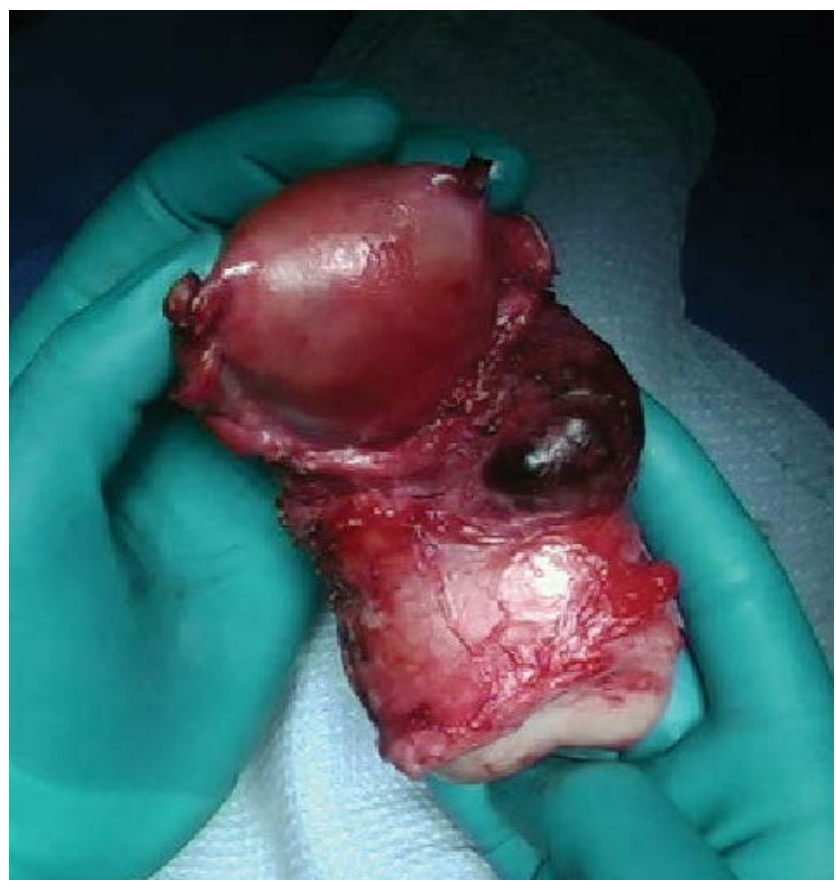

Figure 2: Uterus specimen with placenta increta site at left lower uterine segment. 
$\mathrm{mL}$. She was transported to PACU in stable condition. The patient recovered appropriately during her hospital stay and was discharged on hospital day five in stable condition.

Pathology from the DC\&E procedure revealed products of conception with chorionic villi. Pathology from the exploratory laparotomy revealed dissecting myometrial hematoma with associated necrotic chorionic villi, suggestive of placenta increta or more. Acute salpingitis and serositis of bilateral fallopian tubes. Pathology did not find evidence of a molar pregnancy or definitive uterine perforation.

\section{Discussion}

Our case, in addition to other reported cases of abnormal placental implantation in early pregnancy, supports the idea that invasion of chorionic villi begins at the time of implantation rather than later in pregnancy as a result of absorption of the decidua [3]. Similarly to other reported cases, our patient presented with signs and symptoms consistent with early pregnancy loss and underwent DC\&E procedure at which time severe hemorrhage was encountered. Again, like other reported cases our patient had more than one risk factor for abnormal placentation, most significantly a history of prior cesarean section. In addition to two prior cesarean sections, our patient was of advanced maternal age (AMA).

Molar pregnancy and cesarean scar pregnancy (CSP) were the differential diagnoses considered at the time of the severe hemorrhage. Early ultrasound signs of API are those of CSP, such as low implantation of the gestational sac [6]. Timor-Tritsch, et al. suggested that CSP and $\mathrm{API}$, including early API, are part of a continuum of disease and have similar mechanisms of formation and progression [5]. Due to similar histological presentation, Timor-Tritsch, et al. study showed that it was nearly impossible to differentiate CSP from early API based off pathology. Instead they suggested that early (second trimester) API and CSP are early manifestations of the same entity that are part of a spectrum of disease with morbidly adherent placenta being the most severe. With that said, it was evident that both CSP and API cause significant morbidity and obstetrical complications.

Typically API is diagnosed on second and third trimester scans, however a systematic review showed that ultrasound findings can be found as early as the first trimester [6]. This review showed that $91.4 \%$ of API cases were detected on ultrasound at least once during the pregnancy and of those detected on ultrasound 95.1\% had ultrasound findings before 11 weeks [6]. Ultrasound findings suggestive of API include: Loss of clear zone, placental lacunae, bladder wall interruption, uterovesical hypervascularity, and vascular invasion of parametria [7]. Low anterior implantation of the gestational sac close to or within a previous scar and placenta lacunae were the most common ultrasound findings in early pregnancy $[6,8]$. Cali, et al. study showed at 6-9 weeks ultrasound findings of API were those of CSP, low implantation of the gestational sac within or near the cesarean scar [7]. By 11-14 weeks it is difficult to determine low implantation, however other findings of API may still be present. A retrospective review of this patient's imaging reveals the possibility of the presence of lacunae, hypervascularity and loss of the clear zone. Although devastating, API is still an uncommon obstetrical complication, so it is unclear the role for screening ultrasounds in patients with risk factors. In patients with significant risk factors, there may be benefits to screening ultrasounds, notifying radiologists of increased risk, and having lower thresholds for recommending further imaging, such as MRI. Early detection would be beneficial for better planning and counseling prior to taking a patient to the OR.

Through clarity of hind sight, consulting interventional radiology for UAE may have played a role in this case if implemented earlier in the post-operative period after the initial procedure. Implementation of uterotonics in this setting is not a definitive treatment for patients with hemorrhage when suspecting abnormal placental implantation, molar pregnancy, CSP or cervical pregnancy. The use of the Bakri balloon may have stabilized the hemorrhage initially by applying pressure to the lower uterine segment. However, as the uterine fundus contracted it is likely that the balloon could no longer sit in the proper position to continue to tamponade the bleeding. Early embolization may have acutely stopped the hemorrhage and allowed preservation of the uterus. In the future, it will be important to consider early UAE in the setting of hemorrhage, abnormal ultrasound findings and a high suspicion for CSP, molar pregnancy, cervical scar pregnancy or API. In addition to ending a hemorrhage and decreasing maternal morbidity, UAE can also attempt to preserve future child-bearing.

\section{Conclusion}

Due to indistinguishable histology, it is difficult to say with certainty if this case represents a CSP or an early API. With a history of two cesarean pregnancies and the location of the mass seen in Figure 2, CSP should be considered as a differential diagnosis. Both API and CSP in first and early second trimester are uncommon occurrences but are becoming more common due to increasing rates of cesarean delivery. Due to significant maternal morbidity associated with API, it is important to have a high index of suspicion and proper preparation for patients with multiple risk factors for API. All patients need to be counseled on their risks of API and the consequences associated with this diagnosis $[9,10]$. More studies need to be conducted to determine the role of early ultrasound screening for API and CSP. One may consider more thorough screening in patients with multiple risk factors, including multiple cesarean sections. However, due to a high false positive rate one 
must also take into account anxiety and fear for patients if early screening were to be positive. There should be a low threshold for further imaging in patients with multiple risk factors, especially cesarean sections and abnormal appearing ultrasounds. If faced with a similar presentation it is important to consider, and utilize UAE as an alternative to hysterectomy.

\section{Funding}

This work and manuscript preparation was unfunded.

\section{Conflict of Interest}

There are no conflicts of interest.

\section{References}

1. Gabbe SG (2017) Obstetrics normal and problem pregnancies. Elsevier, Philadelphia, PA.

2. Ecker JL, Sorem KA, Soodak L, Roberts DJ, Safon LE, et al. (1992) Placenta increta complicating a first-trimester abortion. A case report. J Reprod Med 37: 893-895.

3. Papadakis JC, Christodoulou N (2008) Placenta percreta presenting in the first trimester: Review of the literature. Clin Exp Obstet Gynecol 35: 98-102.
4. Behnamfar F, Zafarbakhsh A, Rouholamin S (2014) Placenta increta as an important cause of uterine mass after first-trimester Curettage (case report). Adv Biomed Res 3: 240.

5. Timor-Tritsch IE, Monteagudo A, Cali G, Palacios-Jaraquemada JM, Maymon R, et al. (2014) Cesarean scar pregnancy and early placenta accreta share common histology. Ultrasound Obstet Gynecol 43: 383-395.

6. D'Antonio F, Timor-Tritsch IE, Palacios-Jaraquemada J, Monteagudo A, Buca D, et al. (2018) First-trimester detection of abnormally invasive placenta in high-risk women: Systematic review and meta-analysis. Ultrasound Obstet Gynecol 51: 176-183.

7. Calì G, Timor-Trisch IE, Palacios-Jaraquemada J, Monteaugudo A, Forlani F, et al. (2018) Changes in ultrasonography indicators of abnormally invasive placenta during pregnancy. Int J Gynaecol Obstet 140: 319-325.

8. Yang JI, Kim HY, Kim HS, Ryu HS (2009) Diagnosis in the first trimester of placenta accreta with previous cesarean section. Ultrasound Obstet Gynecol 34: 116-118.

9. Chanrachakul B, Hamontri S, Leopairut J, Herabutya Y (2001) Placenta increta complicating the first trimester abortion. Acta Obstetricia et Gynecologica Scandinavica 80: 467-468.

10. https://www.cdc.gov/nchs/fastats/delivery.htm 